\title{
Intraday dynamics of stock market returns and volatility
}

\author{
Faruk Selçuk $^{\mathrm{a}}$, Ramazan Gençay ${ }^{\mathrm{b}, *}$ \\ ${ }^{a}$ Department of Economics, Bilkent University, Bilkent 06800, Ankara, Turkey \\ ${ }^{\mathrm{b}}$ Department of Economics, Simon Fraser University, 8888 University Drive, Burnaby, British Columbia, V5A 1S6, Canada
}

Available online 18 January 2006

To my friend Faruk who left so early

\begin{abstract}
This paper provides new empirical evidence for intraday scaling behavior of stock market returns utilizing a 5 min stock market index (the Dow Jones Industrial Average) from the New York Stock Exchange. It is shown that the return series has a multifractal nature during the day. In addition, we show that after a financial "earthquake", aftershocks in the market follow a power law, analogous to Omori's law. Our findings indicate that the moments of the return distribution scale nonlinearly across time scales and accordingly, volatility scaling is nonlinear under such a data generating mechanism.
\end{abstract}

(C) 2006 Elsevier B.V. All rights reserved.

Keywords: Intraday return; Intraday volatility; Pivotal statistics; Multifractals; Self-similarity; Scaling; Omori's law

\section{Introduction}

The principle source of the intense intellectual curiosity behind the work on asset pricing is to discover the underlying data generating process of returns (and volatility) and generate testable hypotheses. In general, a hypothesis can always be represented by a model which is a collection of data generating processes. A hypothesis is classified as a simple one if it is represented by a model which contains only one data generating process. If the hypothesis is compound, the model contains more than one data generating process. ${ }^{1}$ In this paper, we examine the nature of the data generating process of returns. Our findings indicate that the return (and volatility) distribution changes nonlinearly under scaling. Furthermore, it is not certain whether the statistical inference from the existing return/volatility literature can be classified as pivotal.

\footnotetext{
*Corresponding author.

E-mail addresses: faruk@bilkent.edu.tr (F. Selçuk), gencay@sfu.ca (R. Gençay).

$U R L:$ http://www.bilkent.edu.tr/ faruk.

${ }^{1} \mathrm{~A}$ hypothesis is classified as a simple one if it is represented by a model which contains only one data generating process. If the hypothesis is compound, the model contains more than one data generating process. Under a compound hypothesis, a test statistic has different distributions under each DGP contained in a model. Therefore, the distribution of the test statistic may not be known if we do not know which DGP generated the data for the model. A test statistic is said to be pivotal only when the distribution of the test statistic under a null hypothesis is the same for all DGP contained in a model. For further discussions of simple and compound hypotheses and the definition of pivotal random variables, the reader may refer to Ref. [1].
} 
The most prominent property of intraday dynamics of returns is the nonlinear scaling of moments across time scales. Also, each moment scales at a different rate (nonlinearly) across each time scale. This prohibits popular continuous time representations, such as Brownian motion, as possible candidates in explaining return dynamics. Since no time scale is privileged, and an arbitrary time scale may not necessarily be representative, any inference based on a particular time scale may need to be interpreted locally without any universal implications.

Surprisingly, different time scales in decision making process of different entities received little attention in economics and finance literature. Studies from the perspective of a universal time clock is the prevalent mode of economic and financial research. In an extensive survey of risk and return trade off in asset pricing, Campbell [2] studies the literature with a universal time clock where the issues in respect to returns, risk and stochastic discount factors are examined. A survey of Ref. [3] looks at stock and bond returns, risk premiums in bond and foreign exchange markets from a universal time scale perspective as well. In a recent survey by Poon and Granger [4], volatility is also examined from a universal time perspective. Our view is that often decisions made in low-frequency time scales act as conditions (restraints) for those decisions which need to be made in higher-frequency scales. Therefore an unconditional universal time scale for the examination of risk and return may not necessarily be the route to follow to examine risk and return. The conditions of risk are not universal across all time scales and this issue deserves careful examination by the theorists by embedding the time scale as a determinant (as a conditioning restraint) in risk-return tradeoffs in dynamic environments. ${ }^{2}$

Dacorogna et al. [6], and Gençay et al. [7-9] argue that conventional time series analysis, focusing exclusively on a time series at a given scale, lacks the ability to explain the nature of the data generating process in general. A process equation that successfully explains daily price changes, for example, is unable to characterize the nature of hourly price changes. On the other hand, statistical properties of monthly price changes are often not fully covered by a model based on daily price changes. Lynch and Zumbach [10] similarly emphasize the importance of a multiscale framework in the analysis of absolute price changes to accommodate the underlying heterogeneity with intraday, daily, weekly and monthly components. Therefore, a comprehensive multi-scale approach is needed to elaborate the market dynamics across time scales in which economic agents operate. ${ }^{3}$

Multifractality is defined by a set of nonlinear restrictions on the moments of the underlying process at different time-scales. Recently, some studies claim that multiscaling reported in the finance literature might be an artifact. For example, Ref. [23] showed that multiscaling can be observed as a result of a slow crossover phenomenon on a finite time event although the underlying process is a monofractal. They warn that it might be hard to distinguish apparent and true multifractal behavior in financial data. Similarly, LeBaron [24] provided a simple stochastic volatility model which is able to produce visual power laws and long memory similar to those from actual return series. However, Stanley and Plerou [25] showed that the simple stochastic volatility model provided by LeBaron [24] cannot produce power laws and long memory. In their model of a multifractal model of asset returns, Mandelbrot et al. and Calvet and Fisher [26-28] demonstrate that most diffusions are characterized by increments that grow locally at a constant rate throughout their sample paths whereas multifractals have multiplicity of local time-varying growth rates. Multifractal models, in general, have the ability to generate nearly uncorrelated returns, with long-memory volatility and meanwhile achieving scale-consistency. ${ }^{4}$

A scale invariant environment is a precursor in our understanding of financial markets and proper management of financial risks. ${ }^{5}$ The primary objective of this paper is to obtain a deeper understanding of the

\footnotetext{
${ }^{2} \mathrm{~A}$ simple example is that often a decision to buy how many cars for a household depends upon the decision made where to live. Buying a home is a lower frequency decision whereas buying and selling cars can be classified as higher frequency decisions. Sharpe [5], for instance, points out the absence of a body of positive microeconomic theory dealing with conditions of risk.

${ }^{3}$ Ghashgaie et al. [11], Mantegna and Stanley [12,13] have analyzed the scaling relationship from the perspective of turbulence in financial markets. Refs. [14-19] examine risk-return relationships across time scales and point out the fragility of inference based on a single universal time scale. A wavelet perspective for time scaling in economics is studied in Refs. [20,21]. Bjornson [22] note the impact of high and low frequency macroeconomic movements in terms of systematic factor risks in an economy.

${ }^{4}$ If the aggregated processes all belong to the same class as the original process, then the underlying framework is referred to as scale consistent.

${ }^{5}$ Ref. [29] has an excellent survey of issues surrounding scaling in finance.
} 
underlying multifractal nature of financial time series towards developing scale invariant models of financial markets. In doing so, we study the scaling laws which govern the data. As noted by Brock [30], scaling laws are useful because "(i) they stimulate the search for interpretive frameworks, (ii) they impose discipline on theory formation (the theory must generate data consistent with the observed scaling results), (iii) (they) give clues to the properties of the space of possible underlying data generating process."

This paper is structured as follows. The following section gives a brief description of data and provides some preliminary analysis including intraday stylized facts. Section 3 reports our findings on the multifractal nature of returns and persistence properties of large shocks. We conclude afterwards.

\section{Data and preliminary analysis}

Our data set is the Dow Jones Industrial Average (DJIA), recorded at five minute (5-min) intervals during the sample period of September 19, 1994-October 16, 2002. ${ }^{6}$ The New York Stock Exchange opens at 13:30 (GMT) and the first record of the DJIA index for that day is registered at 13:35. The market closes at 20:00 (GMT) and the last record of the day is registered at 20:05. ${ }^{7}$ Therefore, there are 79 index records at 5-min intervals during one business day. Overall, our sample period consists of 2,949 days. We eliminated weekends (841 days) and observed holidays (72 days) during which the market was closed. ${ }^{8}$ In addition, we eliminated the days in which there were at least 12 consecutive 5 min zero returns since these periods indicate no market activity (119 days). Note that we eliminated the entire day, not just consecutive zero-return periods during the day, to keep the frequency characteristics of the data set intact. The pre-filtered sample consists of 151,443 data points, covering 1917 business days.

The 5-min stock market return is defined as

$$
r_{t}=\log x_{t}-\log x_{t-1}, \quad t=1,2, \ldots, 151,443,
$$

where $x_{t}$ is the DJIA level at time $t$. The volatility is defined as absolute return, $\left|r_{t}\right|$.

5 -min returns and the corresponding volatility (in percentage) are presented in Fig. 1. The average 5-min return implies a $10.2 \%$ (compound) annual rate of return during the sample period. ${ }^{9}$ The sample statistics indicate that the 5-min stock index return distribution in this frequency is far from being normal. The sample skewness is 0.68 while the sample kurtosis is 55.2, implying several extreme returns relative to the standard normal distribution. Even if the highest and the lowest 500 5-min returns from the sample are excluded, the sample kurtosis is still 5.3. The highest intraday 5 -min positive return $(4.7 \%$ on October 28,1997$)$ is 39 standard deviations $(\sigma)$ away from the mean while the highest intraday 5-min negative return $(2.9 \%$ on October 8,1998 ) is $24 \sigma$. An evaluation of the normal probability density function shows that the probability of observing a large negative return of this size in a normally distributed world would be $10^{-126}$.

In Fig. 2, the estimated autocorrelation coefficients of returns at 5-min intervals are plotted against their lags along with the $95 \%$ Bartlett confidence intervals. There is a significant autocorrelation at the first two lags (10-min), the 42nd lag (half-day) and the 79th lag (one-day). Other seemingly significant autocorrelation coefficients may be due to sampling deviation. The positive autocorrelation for daily stock portfolio returns has long been reported in the literature. ${ }^{10}$ One possible explanation for the first-order positive autocorrelation is the different response time of individual stocks to aggregate information (nonsynchronous trading). A stock market index consists of several stocks with different liquidities. One group of stocks may react to new information more slowly than another group of stocks. Since the autocovariance of a well-diversified portfolio is the average of cross-covariances of individual stocks, significantly positive high-frequency autocorrelations

\footnotetext{
${ }^{6}$ Our results are implicitly conditioned on our choice of 5-min intervals and an alternative would be to study scale effects with data as generated by the market.

${ }^{7}$ See Ref. [31] for an extensive coverage of the market mechanism in the NYSE.

${ }^{8}$ See The New York Stock Exchange official website www.nyse.com for a complete list of observed holidays and other historical closings since 1885 .

${ }^{9}$ The average 5-min return $r_{5}$ is $4.71 \mathrm{e}-006$. Assuming 260 business days in one year, the compound annual rate of return is given by $r_{y}=\left(1+r_{5}\right)^{(79 \times 260)}-1=0.1016$.

${ }^{10}$ See for example, Refs. [32, 33, Ch. 2, 34], and references therein. More recently, Bouchard and Potters [35] have reported significant autocorrelations (up to four lags) of the S\&P500 increments measured at 5-min intervals.
} 

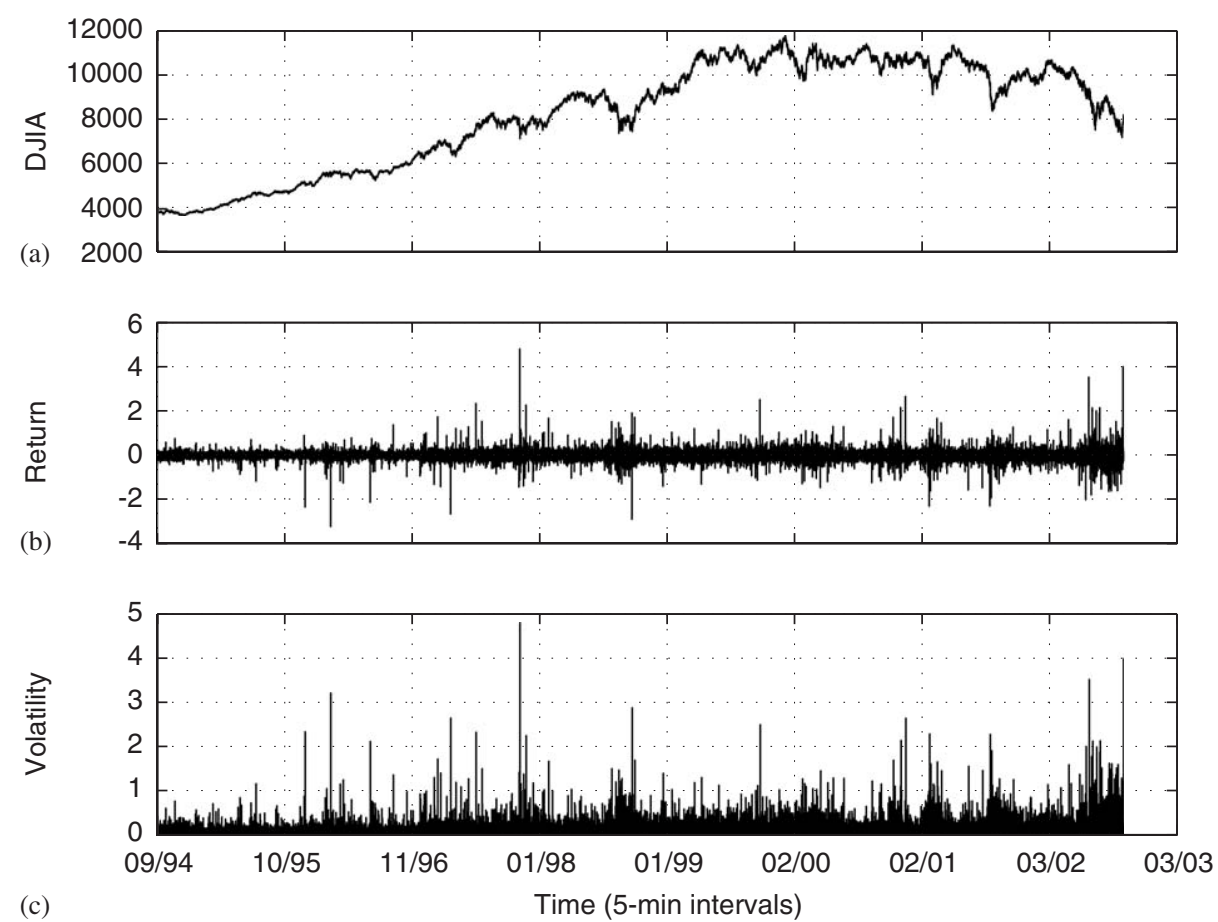

Fig. 1. Dow Jones Industrial Average at 5-min intervals: (a) DJIA level (b) 5-min return (log difference, in percent) (c) 5-min volatility (absolute return, in percent). Sample period is September 19, 1994-October 16, 2002 (151,443 5-min, 1917 days). Data source: Olsen Group (www.olsen.ch).
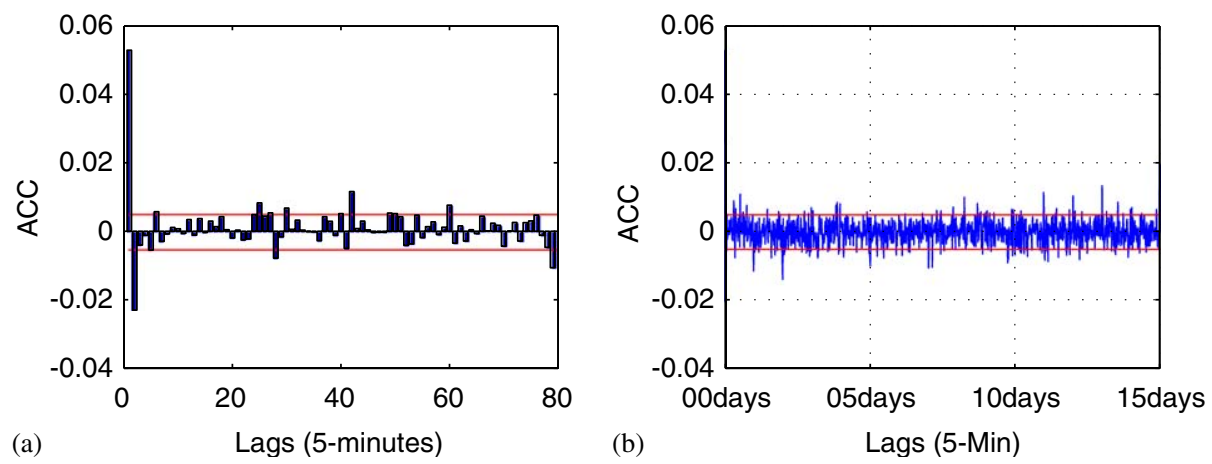

Fig. 2. Autocorrelation coefficients (ACC) of the DJIA returns at 5-min lags. (a) Intraday. (b) 15 days. $95 \%$ confidence intervals are plotted as solid lines. First two, 42nd (half-day) and 79th (one-day) lag autocorrelation coefficients are statistically significant. One business day consists of 795 -min intervals (6 h, $30 \mathrm{~min})$. The sample period is September 19, 1994-October 16, 2002 (151,443 5-min, 1917 days). Data source: Olsen Group (www.olsen.ch).

for stock portfolio returns are feasible [34]. It is well known that bid-ask bounce leads to negative autocorrelation in stock returns [36]. Therefore, one may argue that the true autocorrelation coefficient may be higher than the one reported here.

As illustrated in Fig. 3(a), the sample autocorrelation coefficients of 5-min volatility, defined as absolute returns, are statistically significant, and the intraday autocorrelations have a $\mathrm{U}$ shape pattern. The correlation coefficient takes a value of $23 \%$ at the first lag and decreases afterwards, reaching a minimum of $10 \%$ at around $2.5 \mathrm{~h} \mathrm{lag}$ before starting to rise again. There is a significant peak at lag 79 which indicates that there is a strong seasonal cycle which completes itself in one day. Fig. 3(b) illustrates the autocorrelation coefficients at 

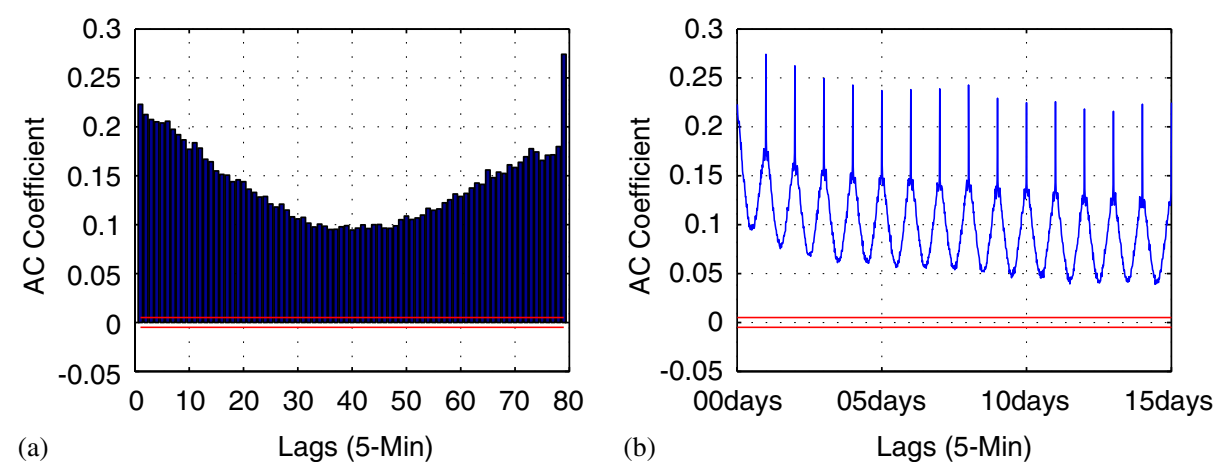

Fig. 3. DJIA volatility autocorrelation coefficients at 5-min lags. (a) Intraday. (b) 15 days. Volatility is defined as absolute percent return. Note that there is a strong daily seasonality. One business day consists of 79 -min intervals ( $6 \mathrm{~h}$ and $30 \mathrm{~min})$. Sample period is September 19, 1994-October 16, 2002 (151,443 5-min, 1917 days). Data source: Olsen Group (www.olsen.ch).
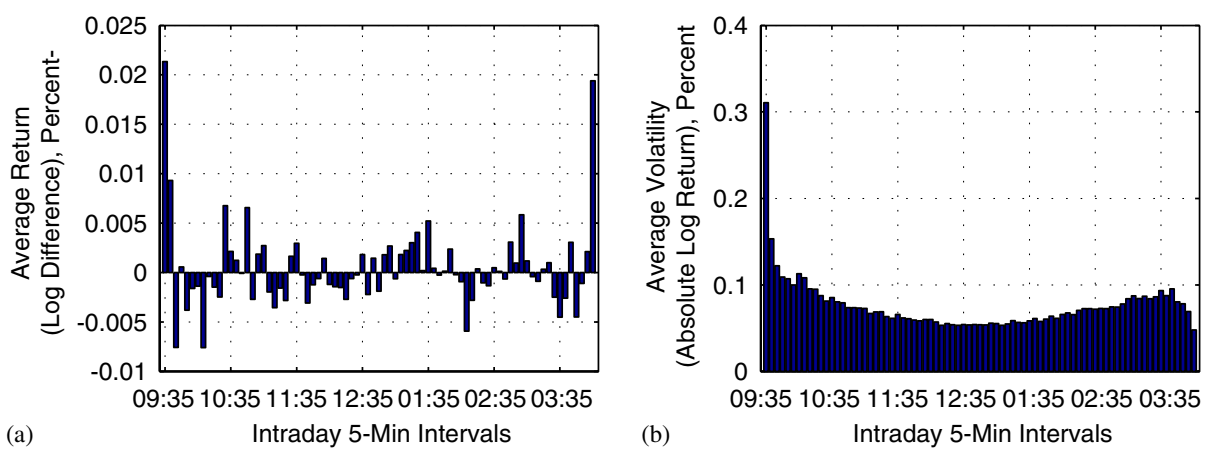

Fig. 4. Average 5-min (a) return (b) volatility during 1-day in sample period. Volatility is defined as absolute percent return. Stock market volatility is relatively high at the opening of the session, especially during the first $45-\mathrm{min}$. Average volatility drops afterwards, reaches to a minimum during lunch hours and slightly increases again. The minimum 5-min average volatility is observed during the last 5-min (closing). Sample period is September 19, 1994-October 16, 2002 (151,443 5-min, 1917 days). Data source: Olsen Group (www.olsen.ch).

5-min lags up to 15 days. Regarding the weekly seasonality, we do not observe a strong peak at 5 days or integer multiple of 5 days. However, this observation should be interpreted with caution since the presence of daily seasonalities may obscure relatively weaker weekly or longer period seasonal dynamics.

Fig. 4 provides 5-min average returns and 5-min average volatilities at 5-min intervals during the day in our sample period. The highest 5 -min returns are observed at the opening and at the closing $5 \mathrm{~min}$. Note that the first 5-min return is actually the return between the previous day's closing and the opening. Therefore, the effects of news and trading when the market is closed should be taken into consideration when interpreting this "first 5-min" return. Recently, Barclay and Hendershott [37] have reported that relatively low after-hours trading can generate significant price discovery, although prices are noisier after hours implying that price discovery is less efficient. They find that the probability of an informed trade is significantly greater during the pre-open than during the post-close, likely due to the accumulated information during nontrading hours. When the market opens, this information advantage is a likely reflection on prices, causing a larger return (and volatility) during the opening than the rest of the day. However, there is no convincing explanation for the large 5-min average return at the closing. Note that the highest average volatilities are observed at the opening of the session, especially during the first 45-min. An early study by Amihud and Mendelson [38] reported that trading during the opening exposes traders to a greater variance than in the close. They attribute this difference to the trading mechanism in the NYSE. The average volatility drops during the early session, reaches a minimum during lunch hours and slightly increases again. Excluding the last 20 min of trading, the volatility has a $U$ shape during the day, similar to the $U$ shape of volatility autocorrelations. The minimum of 
5-min average volatility occurs during the last 5-min before the market closes. The lowest average volatility and a relatively large average return during the closing of the market require further investigation.

\section{Scaling properties}

\subsection{Scaling of risk across time scales}

A fractal is an object which can be subdivided into parts, each of which is a smaller copy of the whole. Selfsimilarity, an invariance with respect to scaling, is an important characteristic of fractals. This means that the underlying object is similar at different scales subject to a scaling factor. A stochastic process, $\left[y_{t}\right]$ is said to be self-similar if for any positive stretching factor $\tau$, the rescaled process with time scale $\tau t, \tau^{-H}\left[y_{t}\right]_{\tau}$, is equal in distribution to the original process $\left[y_{t}\right]$,

$$
\left[y_{t}\right]_{\tau} \stackrel{d}{=} \tau^{H}\left[y_{t}\right] .
$$

The Hurst exponent $H$, also called self-affinity index, or scaling exponent, of $\left[y_{t}\right]$, satisfies $0<H<1$. The operator, $\stackrel{d}{=}$, indicates that the two probability distributions are equal. This necessitates that samplings at different intervals yield the same distribution for the process $\left[y_{t}\right]$ subject to a scale factor. ${ }^{11}$ The principle of scale invariance suggests an observable relationship between volatilities across different time frequencies. Series exhibiting long-term persistence should scale by a factor equivalent to their Hurst exponent which is typically $H>0.5$. $^{12}$ By contrast, a random walk process scales by the factor $H=0.5$. For $0<H<0.5$, the process has a short memory.

Rather than being subject to a unique scaling factor, the underlying data generating process may also follow nonlinear forms of scaling. This is where the concept of multifractals plays an important role in explaining the scaling behavior of several financial time series. In general, we can define an exponent $\xi(q)$ as

$$
E\left(\left|\left[y_{t}\right]_{\tau}\right|^{q}\right)=c(q) \tau^{\xi(q)},
$$

where $E$ is the expectation operator, $q$ is the order of moments, $c(q)$ and $\xi(q)$ are both deterministic functions of $q$. The functions $c(q)$ and $\xi(q)$ are called the scaling functions of the multifractal process. Unifractals or uniscaling are a special case of multifractals where $c(q)$ and $\xi(q)$ are reduced to be linear functions of $q$. For example, $\xi(q)=0.5 q$ for a Gaussian white noise. Multifractal processes, on the other hand, are characterized by the nonlinearity of functions $c(q)$ and $\xi(q)$.

\subsection{Empirical results}

Fractal properties of DJIA returns are investigated by studying the 5-min and aggregate lower frequency intraday returns. The aggregated returns are defined by

$$
\left[r_{t}\right]_{\tau}=\sum_{i=1}^{\tau} r_{\tau(t-1)+i}, \quad t=1, \ldots, 151,443 / \tau,
$$

where $r_{t}$ is the original 5-min returns defined in Eq. (1), $\left[r_{t}\right]_{\tau}$ represents the returns at an aggregated level of $\tau$. For example, the 10-min returns are constructed by summing two 5 -min returns where $\tau=2$. 10-min aggregate returns are defined via

$$
\left[r_{t}\right]_{2}=\sum_{i=1}^{2} r_{2(t-1)+i}, \quad t=1, \ldots, 75,721 .
$$

\footnotetext{
${ }^{11}$ A detailed discussion on self-similarity can be found in Refs. [39-41]. A self-similar process is also called uniscaling (unifractal). A multiscaling (multifractal) process extends the idea of similarity to allow more general scaling functions. Multifractality is a form of generalized scaling that includes both extreme variations and long-memory. Calvet and Fisher [27,28], Matteo et al. [42,43] and Selcuk [18] have recent findings on the evidence of scaling and multifractality in financial markets.

${ }^{12}$ The original work on the Hurst exponent is due to Hurst [44]. Later Lo [45] suggested a modification to eliminate low order persistence.
} 
Similarly, lower frequency intraday returns are obtained for different aggregation periods, $\tau=2$, $3, \ldots, 12,24,36,48,60,72$, corresponding to $10 \mathrm{~min}-6 \mathrm{~h}$ of aggregated returns. The last aggregation period corresponds to approximately one business day since the market is open for $6.5 \mathrm{~h}$ (79 5-min). Thus, the mean moment of absolute returns, for different powers of absolute returns, is examined at 17 time scales, starting 5 -min original returns up to $6 \mathrm{~h}, \tau=1,2,3, \ldots, 12,24,36,48,60,72$. In our estimations we used the following version of Eq. (3)

$$
\left\{E\left(\left|\left[r_{t}\right]_{\tau}\right|^{q}\right)\right\}^{1 / q}=c(q) \tau^{D(q)},
$$

where $D(q)=\xi(q) / q$. We preferred this form so that a fractional Gaussian process (FGN) would result in a constant $D(q)$ for different values of $q$. For example a Gaussian white noise would have $D(q)=0.5$ regardless of the choice of $q$.

To investigate the multifractal properties of DJIA returns, Fig. 5(a) plots $\left\{E\left(\left|\left[r_{t}\right]_{\tau}\right|^{q}\right)\right\}^{1 / q}$ across 17 time scales for different values of $q$ in a double logarithmic scale. The time intervals range from 5 -min to one day. From bottom to top, the values of $q$ increase from 0.5 to 4 at equal increments. The straight lines in the figure indicate the power scaling law of Eq. (5). That is, the $q$ th moment of the returns subject to a scaling factor when moving from a high-frequency interval to low-frequency interval.

The estimated exponent $D(q)$ for different values of $q$ in Eq. (5) is presented in Table 1. In order to obtain robust estimates, we changed the highest aggregation factor $\tau=6,7, \ldots, 12,24,36,48,60,72$ each time and estimated the exponent $D(q)$ for each aggregation period for different values of $q$. The final estimate is obtained as an average of these estimated exponents. In addition, we calculated bootstrapped estimates and their corresponding confidence intervals. The bootstrap is implemented by sampling without replacement from the return data set to produce a new data set each time and from this new sample, new estimates are calculated as an average of changing aggregation period estimates as before. The procedure is replicated 100 times. Reported drift exponents in Table 1 are averages of these bootstrap estimates. 95\% confidence intervals from the bootstrapping results are reported in brackets under each exponent estimate. In order to check the robustness of this procedure, we generated a Gaussian white noise $(D(q)=0.50)$ with a sample size identical to our sample (151,443 data points). Estimated exponents using the same procedure (changing aggregation periods and 100 bootstrapping) for this simulated series in Table 1 show that the procedure produces very reliable estimates.

In Fig. 5(a), each line describes the rate at which moments scale in double logarithmic scale. For all $q$, the scaling is linear and slopes are smaller for higher moments. The interpretation is that the lower the frequency, the higher the value of the corresponding moment from 5-min to 6-h time scales. The basic idea about scaling is that the different moments at a given time scale can be extended to another time scale. For example, the normalized first moment (the mean absolute return) of 5-min absolute returns can be rescaled to the first moment of 6-h absolute returns by considering the scaling factor via $E\left[\left|r_{t}\right|\right]_{72}=1 \times 72^{0.567}=11.3$ (assuming
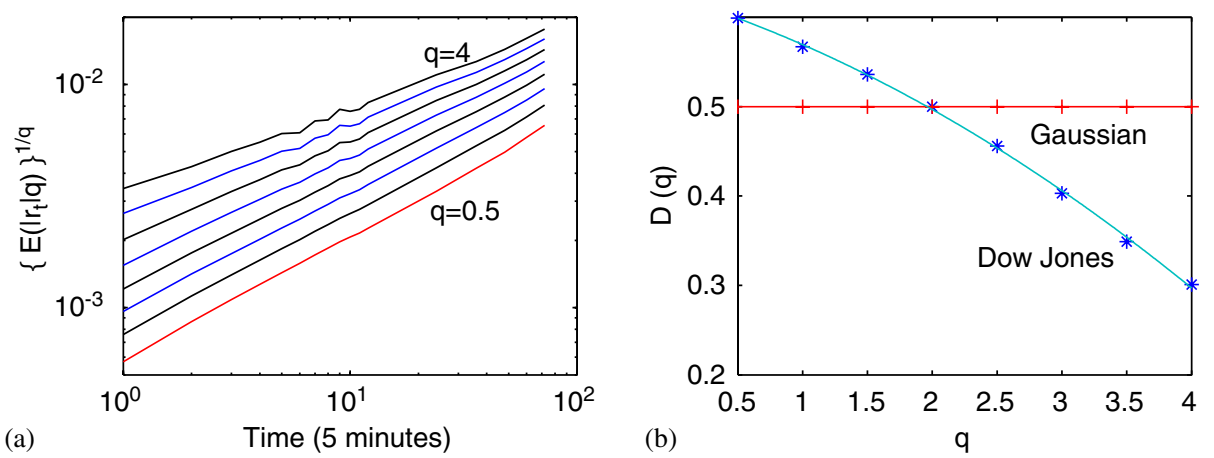

Fig. 5. Intraday scaling. Left: $\left\{E\left(\left|\left[r_{t}\right]_{\tau}\right|^{q}\right)\right\}^{1 / q}$ (vertical axis) against the different time scales $\tau$ (horizontal axis) for different values of the exponent $q=0.5,1,1.5,2,2.5,3,3.5,4$ (from top to bottom) on a log-log scale. $\tau$ values range from 10-min to $6 \mathrm{~h}$. Right: Estimated exponents $D(q)$ are plotted against corresponding order of moments, $q$. A second order estimated polynomial is imposed on this plot. As a benchmark, estimated exponents from a simulated Gaussian white noise for different order of moments are also plotted. Sample period is September 19, 1994-October 16, 2002 (151,443 5-min, 1917 days). Data source: Olsen Group (www.olsen.ch). 
Table 1

Estimated exponents $D(q)$ in Eq. (5) and 95\% bootstrap confidence intervals for the 5-min DJIA returns. As a benchmark, estimated exponents from a simulated Gaussian white noise for different order of moments and $95 \%$ confidence intervals are also provided. Sample period is September 19, 1994-October 16, 2002 (151,443 5-min, 1917 days). Data source: Olsen Group (www.olsen.ch)

\begin{tabular}{|c|c|c|}
\hline$q$ & Dow Jones & Simulated FGN \\
\hline \multirow[t]{2}{*}{0.5} & 0.599 & 0.50 \\
\hline & {$\left[\begin{array}{ll}0.595 & 0.603\end{array}\right]$} & {$\left[\begin{array}{ll}0.494 & 0.504\end{array}\right]$} \\
\hline \multirow[t]{2}{*}{1} & 0.567 & 0.50 \\
\hline & {$\left[\begin{array}{ll}0.563 & 0.570\end{array}\right]$} & {$\left[\begin{array}{ll}0.496 & 0.506\end{array}\right]$} \\
\hline \multirow[t]{2}{*}{1.5} & 0.536 & 0.50 \\
\hline & {$\left[\begin{array}{ll}0.531 & 0.540\end{array}\right]$} & {$\left[\begin{array}{ll}0.495 & 0.504\end{array}\right]$} \\
\hline \multirow[t]{2}{*}{2} & 0.50 & 0.50 \\
\hline & {$\left[\begin{array}{ll}0.496 & 0.503\end{array}\right]$} & {$\left[\begin{array}{ll}0.496 & 0.506\end{array}\right]$} \\
\hline \multirow[t]{2}{*}{2.5} & 0.456 & 0.50 \\
\hline & {$\left[\begin{array}{ll}0.451 & 0.461\end{array}\right]$} & {$\left[\begin{array}{ll}0.495 & 0.504\end{array}\right]$} \\
\hline \multirow[t]{2}{*}{3} & 0.403 & 0.50 \\
\hline & {$\left[\begin{array}{lll}0.397 & 0.411]\end{array}\right.$} & {$\left[\begin{array}{ll}0.495 & 0.505\end{array}\right]$} \\
\hline \multirow[t]{2}{*}{3.5} & 0.349 & 0.50 \\
\hline & {$\left[\begin{array}{lll}0.339 & 0.357\end{array}\right]$} & {$\left[\begin{array}{ll}0.495 & 0.505\end{array}\right]$} \\
\hline \multirow[t]{2}{*}{4} & 0.30 & 0.50 \\
\hline & {$\left[\begin{array}{ll}0.286 & 0.312\end{array}\right]$} & {$\left[\begin{array}{ll}0.495 & 0.504\end{array}\right]$} \\
\hline
\end{tabular}

5-min mean absolute return is $1 \%$ ). Similarly, the normalized second moment of 5-min absolute returns can be utilized to obtain the second moment of 6-h absolute returns as $E\left|r_{t}\right|_{72}^{2}=72^{2 \times 0.50}$. For the DJIA series, the 5min mean absolute return is $0.076 \%$. For $D(1)=0.567$, the 6 -h absolute return is $0.076 \times 72^{0.567}=0.86 \%$. If we examine actual data (returns aggregated at 72 5-min, 6-h scale), the actual mean absolute return at 6-h scale is $0.81 \%$. Note that with square root scaling $(D(q)=0.5)$, the 6 -h absolute return would only be $0.64 \%$ $\left(0.076 \times 72^{0.5}\right)$. The study of the first and the last one-thirds of the data do not change the scaling results as illustrated in Fig. 6.

The estimation results indicate different exponents $D(q)$ for different values of $q$ in Eq. (5), which suggest that there are different scaling laws for different order of moments. The lower moments of absolute returns scale faster than the higher moments. Particularly, the moments up to $q=2$ scale faster than a Gaussian white noise while the moments greater than $2(q>2)$ scale slower than a Gaussian white noise. The second moment $q=2$ appears to be the borderline. For example, suppose that the normalized mean absolute return is $1 \%$. If we assume a Gaussian white noise, the corresponding 6-h mean absolute return would be $72^{0.50}=8.5 \%$ while the estimated exponent implies $72^{0.567}=11.3 \%$ mean absolute return at the 6 -h scale. Note that higher moments of the absolute returns give more weight to large observations (tails of the empirical distribution). Similar results are obtained earlier in the literature. By employing Eq. (5) in their estimations, Dacorogna et al. [6] report $D(1)$ around 0.60 and $D(2)$ at around 0.50 for major foreign exchanges and Eurofutures. Similarly, Selçuk [18] reports that the estimated $D(1)$ is in between 0.55 and $0.59, D(2)$ is around 0.50 and the higher moments have $D(q)$ less than 0.50 .

Recall that the return process is monofractal if $\xi(q)=q \xi(1)$ is a linear function of $q$ and multifractal if $\xi(q)$ is nonlinear function of $q$. To illustrate, the estimated exponent $D(q)$ is plotted against $q$ along with estimated $D(q)$ from a Gaussian white noise in Fig. 5(b). The exponent $D(q)$ for the Gaussian white noise stays constant at 0.5 as expected while the estimated exponents from 5-min Dow Jones return series start with 0.6 at $q=0.5$ and fall to 0.3 at $q=4$, suggesting multifractal behavior of the return process. An estimated second order polynomial for the estimated exponents $D(q)$ in Fig. 5(b) is

$$
y=-0.0023 q^{2}-0.023 q+0.62
$$

where $y$ is the estimated exponent. Clearly, $D(q)$ is nonlinear and the nonlinearity of $D(q)$ verifies that the DJIA return process is multifractal. 

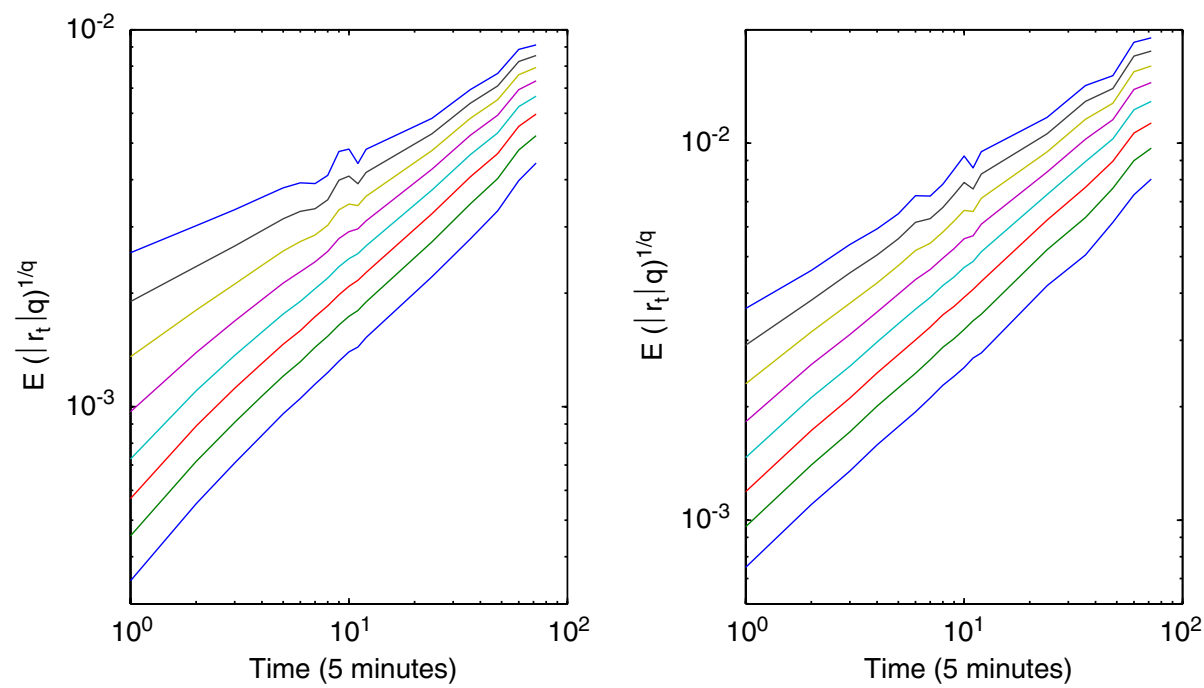

Fig. 6. Intraday scaling. Left: September 19, 1994-December 31, 1996, Right: January 2, 2000-October 16, 2002 in 5-min frequency. $\left\{E\left(\left|\left[r_{t}\right]_{\tau}\right|^{q}\right)\right\}^{1 / q}$ (vertical axis) against the different time scales $\tau$ (horizontal axis) for different values of the exponent $q=$ $0.5,1,1.5,2,2.5,3,3.5,4$ (from top to bottom) on a $\log$ - $\log$ scale. $\tau$ values range from 10-min to $6 \mathrm{~h}$. Data source: Olsen Group (www.olsen.ch).
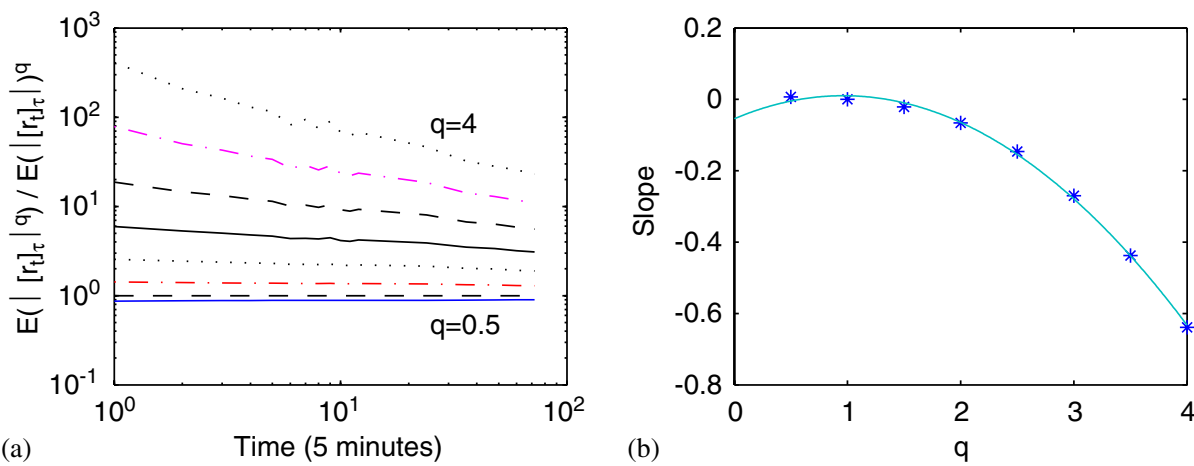

Fig. 7. Intraday scaling. Left: $E\left(\left|\left[r_{t}\right]_{\tau}\right|^{q}\right) / E\left(\left|\left[r_{t}\right]_{\tau}\right|\right)^{q}$ (vertical axis) against the different time intervals $\tau$ (horizontal axis) for different values of the exponent $q=0.5,1,1.5,2,2.5,3,3.5,4$ (from bottom to top) on a log-log scale. Right: Estimated slopes from the left panel against corresponding exponent, $q$. A second order polynomial fit is imposed on this plot. Sample period is September 19, 1994-October 16, 2002 (151,443 5-min, 1917 days). Data source: Olsen Group (www.olsen.ch).

The nonlinearity of $\xi(q)$ may also be tested by plotting $E\left(\left|\left[r_{t}\right]_{\tau}\right|^{q}\right) / E\left(\left|\left[r_{t}\right]_{\tau}\right|\right)^{q}$ versus time intervals for different values of $q$. Fig. 7(a) shows that $E\left(\left|\left[r_{t}\right]_{\tau}\right|^{q}\right) / E\left(\left|\left[r_{t}\right]_{\tau}\right|\right)^{q}$ is an approximate linear function of $\tau$ for different values of $q$ ranging from 0.5 (bottom) to 4 (top) in a log-log space. The straight lines which are observed have a slope of $\xi(q)-q \xi(1)$. In the case that $\xi(q)$ is a linear function of $q$, the slopes of the straight lines must be 0 . However, if $\xi(q)$ is nonlinear, a trend should be expected. The graph shows that the slopes of the straight lines are nonzero except for $q=1$. When $q<1$, the slope is positive. In other cases, negative slopes are observed. To illustrate the properties of the exponent function, the estimated slopes from Fig. 7(a) are plotted against corresponding exponents, $q$, in Fig. 7(b). The estimated second order polynomial in this case is

$$
y=-0.07 q^{2}+0.13 q-0.054,
$$


where $y$ is the estimated slope coefficient. Once again, it is evident that $\xi(q)$ is a nonlinear function of $q$, which provides further evidence that the DJIA return process is multifractal.

\subsection{Financial earthquakes and aftershocks}

Mandelbrot [46,47] eloquently demonstrated the importance of Pareto's law for the power decay of the tails of return distributions. ${ }^{13}$ Although the tails of return distributions follow a power law at high frequency scales, this decay does not take the time order into account. The time path followed by these shocks is an identifying factor for a model of returns. Along with Sornette et al. [49], Lillo and Mantegna [50,51] and Selçuk [18], we examine the persistence of shocks in intraday scales.

A major earthquake in a region is usually followed by smaller ones, labeled as "aftershocks". There are several approaches to describe the dynamics of aftershocks. A well-known simple rule is the Gutenberg-Richter relation, which says that the number of earthquakes of magnitude $M$ or greater, $N(M)$, is given by

$$
\log _{10} N(M)=a-b M,
$$

where $a$ and $b$ are two constants. In several studies, $b$ is found to be within the range of 0.7 to 1 regionally. However, for larger geographical areas and the world, the slope parameter is usually 1 . The interpretation is such that we will observe approximately ten times as many aftershocks with a magnitude one unit less than the main shock. Fitting the tail of a distribution in finance is analogous to this relationship.

Another approach relates the time after the main shock to the number of aftershocks per unit time, $n(t)$. This is known as the Omori law. ${ }^{14}$ Omori's law states that the number of aftershocks per unit time decays according to the power law of $t^{-p}$. In order to avoid divergence at $t=0$, Omori's law is rewritten as

$$
n(t)=K(t+\tau)^{-p},
$$

where $K$ and $\tau$ are constants. By integrating Eq. (7) between 0 and $t$, the cumulative number of aftershocks between the main shock and the time $t$ can be expressed as

$$
N(t)=K\left[(t+\tau)^{1-p}-\tau^{1-p}\right] /(1-p),
$$

when $p \neq 1$ and $N(t)=K \ln (t / 1+\tau)$ for $p=1[50]$.

By performing numerical simulations and theoretical modeling, Lillo and Mantegna [50] show that the nonlinear behavior observed in real market crashes cannot be described by popular volatility models. Particularly, they show that simulated $\operatorname{GARCH}(1,1)$ time series converges to its stationary phase very quickly after a large shock and it is unable to show a significant nonlinear behavior. Recently, a series of papers has investigated the behavior of volatility in financial markets after big crashes. An early work by Sornette et al. [49] shows that the implied volatility in the S\&P500 after the 1987 financial crash has a power law-periodic decay. Lillo and Mantegna [50,51] have shown that S\&P500 index returns above a large threshold are well described by a power law function which is analogous to Omori's law in Eq. (8). More recently, Selçuk [18] has investigated the financial aftershocks (defined as absolute daily returns above a certain threshold following a significant drop in the stock market) in ten different emerging economies. Selçuk [18] has reported that the majority of estimated exponents in Eq. (8) for these economies is less than 1, concentrating between 0.50 and $0.70 .^{15}$

On October 8, 1998, the DJIA went down from 7827.9 at 9:40 AM to 7606.3 at 9:45 AM resulting in a 2.9\% drop within $5 \mathrm{~min}$ and the trade was not suspended. This shock is the largest percentage drop in 5-min in our sample without an interruption in the market. Fig. 8 plots the cumulative number of aftershocks for the following 23705 -min intervals (dotted line). An aftershock is defined as 5-min absolute return greater than $3 \sigma$ where $\sigma=0.0012$ is the 5 -min sample standard deviation. The solid line is the best fit of Eq. (8) for the sample. The estimated exponent $p$ is 0.95 . Another example is provided in Fig. 9. On January 3, 2001, the DJIA went up from 10636 at 1:15 PM to 10920 at 1:20 PM resulting in a $2.6 \%$ increase in $5 \mathrm{~min}$. This shock is one of the

\footnotetext{
${ }^{13}$ LeBaron [24], Mandelbrot [48] Stanley and Plerou [25] discuss whether stochastic volatility models follow a power law behavior.

${ }^{14}$ See Refs. [52,50,51].

${ }^{15}$ Refs. [53,54] show that the exponent of the "Omori" law (measured differently) is a function of the size of the peak, which can be predicted by the multifractal random walk model.
} 


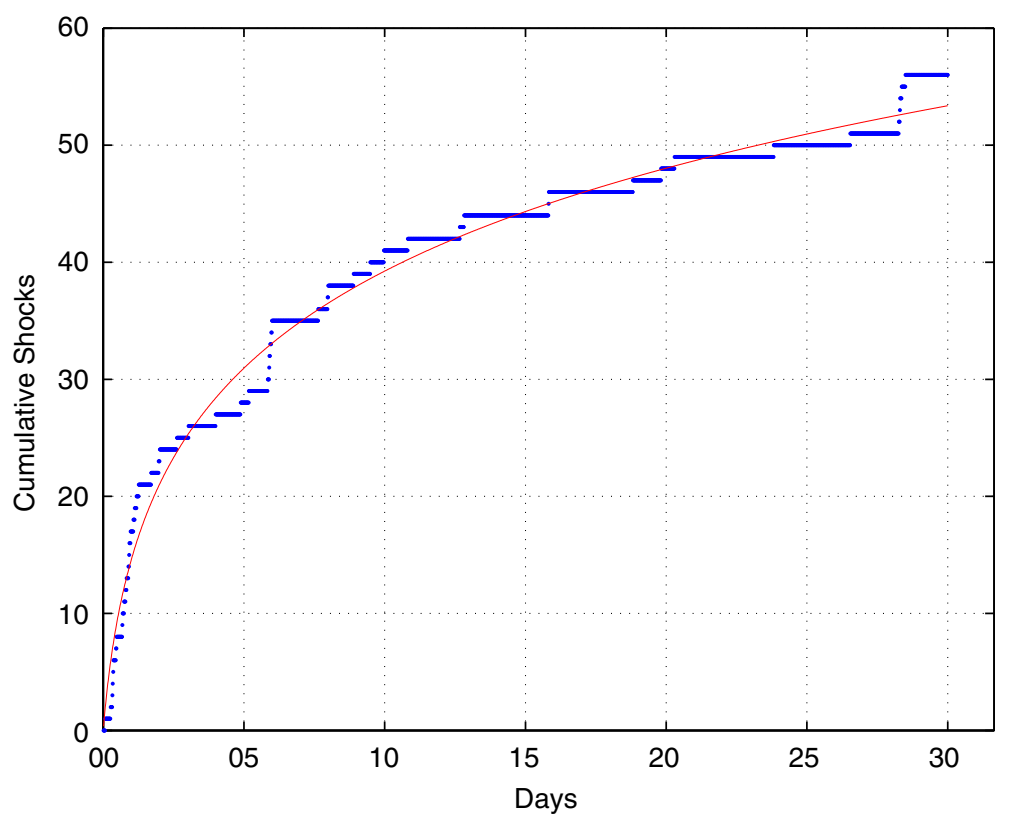

Fig. 8. Cumulative number of aftershocks and Omori's Law. On October 8, 1998, DJIA went down from 7827.9 at 9:40 AM to 7606.3 at 9:45 AM resulting in a 2.9\% drop within $5 \mathrm{~min}$ and the trade was not suspended. This shock is the largest percentage drop in a 5-min in our sample without an interruption in the market. The plot shows the cumulative number of aftershocks for the following 23705 -min intervals (30 business days) (dotted line). An aftershock is defined as 5-min absolute return greater than $3 \sigma$ where $\sigma=0.0012$ is the 5-min sample standard deviation. The solid line is the best fit of Eq. (8) for the above sample. The estimated exponent $p$ is 0.95 . Data source: Olsen Group (www.olsen.ch).

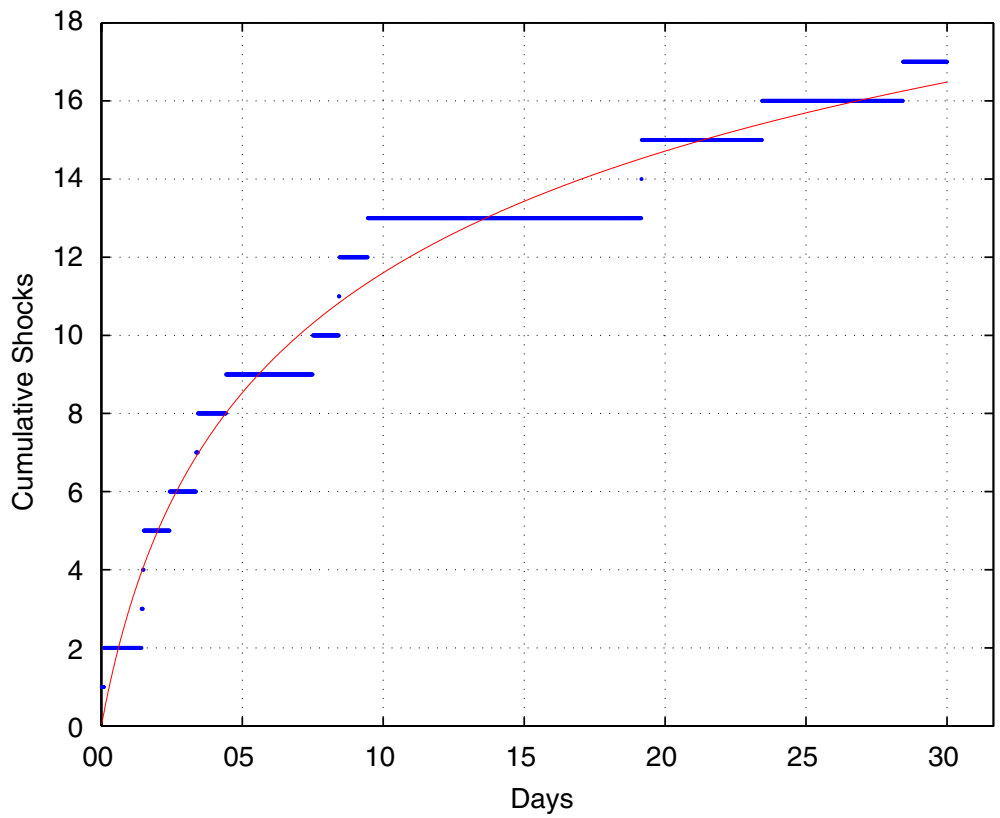

Fig. 9. Cumulative number of aftershocks and Omori Law. On January 3, 2001, DJIA went up from 10636 at 1:15 PM to 10920 at 1:20 PM resulting in a $2.6 \%$ increase in within 5 min. This shock is one of the largest percentage increases in a 5 -min during the day in our sample without an interruption in the market. The plot shows the cumulative number of aftershocks for the following 23705 -min intervals (30 business days). An aftershock is defined as 5-min absolute return greater than $3 \sigma$ where $\sigma=0.0012$ is the 5-min sample standard deviation. The solid line is the best fit of Eq. (8) for the above sample. The estimated exponent $p$ is 1.17. Data source: Olsen Group (www.olsen.ch). 
largest percentage increase in 5-min during the day in our sample without an interruption in the market. Fig. 9 illustrates the cumulative number of aftershocks for the following 2370 5-min intervals (30 business days). As before, an aftershock is defined as the 5-min absolute return greater than $3 \sigma$ where $\sigma=0.0012$. The estimated exponent $p$ is 1.17 and Eq. (8) describes intraday aftershock dynamics fairly well.

\section{Conclusions}

This paper provides new empirical evidence for intraday scaling behavior of stock market returns utilizing a 5-min stock market index (the Dow Jones Industrial Average) from the New York Stock Exchange. Our findings indicate that the return (and volatility) distribution changes nonlinearly under scaling. Furthermore, it is not certain whether the statistical inference from the existing return/volatility literature can be classified as pivotal.

\section{Acknowledgment}

Faruk Selçuk gratefully acknowledges the financial support from the Research Development Grant Program of Bilkent University. Ramazan Gençay gratefully acknowledges financial support from the Natural Sciences and Engineering Research Council of Canada and the Social Sciences and Humanities Research Council of Canada. We are grateful to Olsen Group (www. olsen. ch), Switzerland for providing the data. My co-author Faruk Selçuk passed away during the process of writing this paper. I take responsibility for all errors.

\section{References}

[1] R. Davidson, J.G. MacKinnon, Econometric Theory and Methods, Oxford Press, New York, 2003.

[2] J.Y. Campbell, Asset pricing at the millennium, J. Finance 4 (2000) 1515-1567.

[3] J.H. Cochrane, New facts in finance, Federal Reserve Bank of Chicago 23 (1999) 59-78.

[4] S. Poon, C.W.J. Granger, Forecasting volatility in financial markets: a review, J. Econ. Literature 41 (2003) $478-539$.

[5] W.F. Sharpe, Capital asset prices: a theory of market equilibrium under conditions of risk, J. Finance 19 (1964) $425-442$.

[6] M. Dacorogna, R. Gençay, U. Muller, R. Olsen, O. Pictet, An Introduction to High-Frequency Finance, Academic Press, San Diego, 2001.

[7] R. Gençay, G. Ballocchi, M. Dacorogna, R. Olsen, O. Pictet, Real-time trading models and the statistical properties of foreign exchange rates, Int. Econ. Rev. 43 (2002) 463-491.

[8] R. Gençay, F. Selçuk, B. Whitcher, Asymmetry of information flow between volatilities across time scales, manuscript, 〈http:// www.sfu.ca/ rgencay/whmm.pdf (2003).

[9] R. Gençay, M. Dacorogna, R. Olsen, O. Pictet, Real-time trading models and market behavior, J. Econ. Dyn. Control 27 (2003) 909-935.

[10] P.E. Lynch, G.O. Zumbach, Market heterogeneities and the causal structure of volatility, Quant. Finance 3 (2003) $320-331$.

[11] S. Ghashgaie, W. Breymann, J. Peinke, P. Talkner, Y. Dodge, Turbulent cascades in foreign exchange markets, Nature 381 (1996) 767-770.

[12] R.N. Mantegna, H.E. Stanley, Turbulent and exchange markets, Nature 383 (1996) 587-588.

[13] R.N. Mantegna, H.E. Stanley, Stock market dynamics and turbulence: parallel analysis of fluctuation phenomena, Physica A 239 (1997) 255-266.

[14] A. Arnéodo, J.F. Muzy, D. Sornette, Direct causal cascade in the stock market, Eur. Phys. J. B 2 (1998) $277-282$.

[15] R. Gençay, F. Selçuk, B. Whitcher, An Introduction to Wavelets and Other Filtering Methods in Finance and Economics, Academic Press, San Diego, 2001.

[16] R. Gençay, F. Selçuk, B. Whitcher, Multiscale systematic risk, J. Int. Money Finance 24 (2005) 55-70.

[17] R. Gençay, F. Selçuk, B. Whitcher, Systematic risk and time scales, Quant. Finance 3 (2003) 108-116.

[18] F. Selçuk, Financial earthquakes, aftershocks and scaling in emerging stock markets, Physica A 333 (2004) 306-316.

[19] Z. Xu, R. Gençay, Scaling, self-similarity and multifractality in FX markets, Physica A 323 (2003) 578-590.

[20] J.B. Ramsey, The contribution of wavelets to the analysis of economic and financial data, Phil. Trans. R. Soc. London A 357 (1999) 2593-2606.

[21] J.B. Ramsey, Wavelets in economics and finance: past and future, Stud. Nonlinear Dyn. \& Econometrics 3 (2002) 1.

[22] B. Bjornson, H.S. Kim, K. Lee, Low and high frequency macroeconomic forces in asset pricing, Quart. Rev. Econ. Finance 39 (1999) 77-100.

[23] J.P. Bouchaud, M. Potters, M. Meyer, Apparent multifractality in financial time series, Eur. Phys. J. B 13 (2000) $595-599$. 
[24] B. LeBaron, Stochastic volatility as a simple generator of apparent financial power laws and long memory, Quant. Finance 1 (2001) 621-631.

[25] H.E. Stanley, V. Plerou, Scaling and universality in economics: Empirical results and theoretical interpretation, Quant. Finance 1 (2001) 563-567.

[26] B. Mandelbrot, A. Fisher, L. Calvet, A multifractal model of asset returns, cowles Foundation Discussion Paper No. 1164, Yale University, 1997.

[27] L. Calvet, A. Fisher, Forecasting multifractal volatility, J. Econometrics 105 (2001) 27-58.

[28] L. Calvet, A. Fisher, Multifractality in asset returns: theory and evidence, Rev. Econ. Stat. 84 (2002) 381-406.

[29] J.D. Farmer, Physicists attempt to scale the ivory towers of finance, Computing in Science and Engineering (IEEE) November/ December (1999) 26-39.

[30] W.A. Brock, Scaling in economics: a reader's guide, Ind. Corporate Change 8 (1999) 409-446.

[31] L. Harris, Trading \& Exchanges: Market Microstructure for Practitioners, Oxford University Press, Oxford, 2003.

[32] M. O'Hara, Market Microstructure Theory, Blackwell Publishers Ltd, Oxford, 1995.

[33] J.Y. Campbell, A.W. Lo, A.C. MacKinlay, The Econometrics of Financial Markets, Princeton University Press, Princeton, NJ, 1997.

[34] D. Ahn, J. Boudoukh, M. Richardson, R.F. Whitelaw, Partial adjustment or stale prices? Implications from stock index and futures return autocorrelations, Rev. Financial Stud. 15 (2002) 655-689.

[35] J.-B. Bouchaud, M. Potters, Theory of Financial Risk and Derivative Pricing: From Statistical Physics to Risk Management, Cambridge University Press, Cambridge, 2003.

[36] R. Roll, A simple implicit measure of the effective bid-ask spread in an efficient market, J. Finance 39 (1984) $1127-1139$.

[37] M.J. Barclay, T. Hendershott, Price discovery and trading after hours, Rev. Financial Stud. 16 (2003) $1041-1073$.

[38] Y. Amihud, H. Mendelson, Trading mechanisms and stock returns: an empirical investigation, J. Finance 42 (1986) $533-553$.

[39] B.B. Mandelbrot, Fractals, Form, Chance and Dimension, W.H. Freeman and Company, New York, 1977.

[40] B.B. Mandelbrot, The Fractal Geometry of Nature, W.H. Freeman and Company, New York, 1983.

[41] J. Beran, Statistics for Long-Memory Processes, Chapman \& Hall, London, 1994.

[42] T.D. Matteo, T. Aste, M.M. Dacorogna, Scaling behaviors in differently developed markets, Physica A 324 (2003) $183-188$.

[43] T.D. Matteo, T. Aste, M.M. Dacorogna, Using the scaling analysis to characterize financial markets, J. Empirical Finance, 2004, forthcoming.

[44] H.E. Hurst, Long-term storage capacity of reservoirs, Trans. Am. Soc. Civil Engineers 116 (1951) 770-799.

[45] A.W. Lo, Long-memory in stock market prices, Econometrica 59 (1991) 1279-1313.

[46] B.B. Mandelbrot, New methods in statistical economics, J. Political Econ. 71 (1963) 421-440.

[47] B.B. Mandelbrot, The variation of certain speculative prices, J. Business 36 (1963) 394-419.

[48] B.B. Mandelbrot, Stochastic volatility, power laws and long memory, Quantitative Finance 6 (2001) 558-559.

[49] D. Sornette, A. Johansen, J.P. Bouchaud, Stock market crashes, precursors and replicas, J. Phys. I 6 (1996) 167-175.

[50] F. Lillo, R.N. Mantegna, Dynamics of financial market index after a crash, Manuscript, http://arXiv.org/abs/cond-mat/0209685 (2002).

[51] F. Lillo, R.N. Mantegna, Power law relaxation in a complex system: Omori law after a financial market crash, Phys. Rev. E 68 (2003) $1-5$.

[52] F. Omori, On the after-shocks of earthquakes, J. Coll. Sci. Imp. Univ. Tokyo 7 (1894) 111-200.

[53] D. Sornette, Y. Malevergne, J.F. Muzy, What causes crashes?, Risk 16 (2003) 67-71.

[54] D. Sornette, A. Helmstetter, Endogeneous versus exogeneous shocks in systems with memory, Physica A 318 (2003) 577-591. 